J Neurol Neurosurg Psychiatry 2005;76(Suppl V):v2-v7. doi: 10.1136/jnnp.2005.082867

r ementia refers to a syndrome that is characterised by progressive deterioration of cognitive functions. Neuropsychiatric symptoms, such as apathy, agitation, and depression, are also common. With increasing loss of function, a patient is gradually robbed of his or her independence. Eventually, placement in a nursing home may be necessary. Patients with dementia usually survive 7-10 years after onset of symptoms. Dementia places a tremendous burden not only on caregivers, but also on society, and has already been established as one of the major challenges of this century. ${ }^{1}$

Epidemiology refers to the medical science that studies frequencies of disease. ${ }^{2}$ Measures of frequency that are often used in epidemiology are prevalence and incidence. The concept of prevalence refers to the number of patients with a disease at a certain moment in time, whereas measures of incidence reflect the number of new cases over time. Although important for health care planners, the knowledge of frequency of disease in itself is not the goal of epidemiology. Rather, the aim is to gain insight into the mechanisms that cause disease, eventually to be able to cure or prevent disease. Therefore, frequencies are studied in relation to determinants, or risk factors. Although marked as "the epidemic of our century", still surprisingly little is known about the epidemiology of dementia. In this chapter, a brief overview will be given of the epidemiology and risk factors of dementia. Furthermore, we comment on some specific methodological problems associated with studies in dementia.

\title{
SYNDROME AND DISEASE
}

The syndrome of dementia may be caused by various underlying diseases, each characterised by a specific constellation of signs and symptoms in combination with a presumed underlying substrate of neuropathology (fig 1). Alzheimer's disease (AD) is the most prevalent cause of dementia. It is a neurodegenerative disorder, generally assumed to be caused by neuritic plaques and neurofibrillary tangles accumulating in the brain. The second most prevalent cause of dementia is vascular dementia $(\mathrm{VaD})$, which may be caused by various types of vascular pathology in the brain, such as "large vessel" (large territorial or strategical infarctions) and "small vessel" (lacunes and white matter hyperintensities) disease. Other frequent causes of dementia include frontotemporal lobar degeneration and dementia with Lewy bodies. It is often difficult (if not impossible) to reliably distinguish between subtypes of dementia (we will come back to this subject in the section on methodological issues). Therefore, epidemiological studies often focus on dementia as a whole, sometimes giving separate numbers for the two most important subtypes-AD and VaD.

\section{PREVALENCE}

Prevalence is defined as the proportion of a population that has disease at a specific point in time. Prevalence estimates vary highly between studies. These variations may be due to variations in study population-that is, reflect real differences. For example, age is the most important risk factor for dementia. Differences in age between populations will result in different estimates of prevalence. Alternatively, and just as plausible, is the assumption that differences in prevalence estimates are caused by methodological differences, such as study design and diagnostic procedure. One solution to obtain more certainty about the value of prevalence is to combine data from multiple studies in a meta-analysis. Meta-analyses have two advantages. First, small differences due to methodological differences between studies level out. Secondly, and more importantly, the analysis is based on a far larger sample than a single study could ever realise, resulting in more precise estimates.

\section{Pooled estimates of prevalence}

In 2000, prevalence data from 11 European population based studies were pooled to obtain stable estimates of prevalence of dementia in the elderly $(>65$ years $){ }^{3}$ Age standardised prevalence was $6.4 \%$ for dementia (all causes) , $4.4 \%$ for $\mathrm{AD}$, and $1.6 \%$ for VaD. Prevalence of dementia was higher 


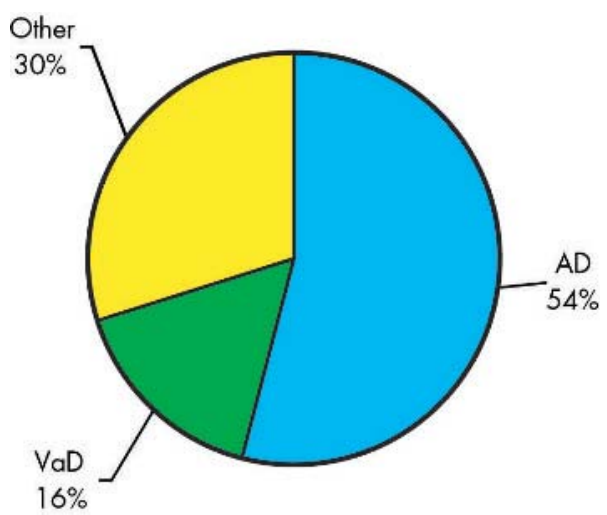

Figure 1 Causes of dementia with late onset ( $\geqslant 65$ years). $A D$, Alzheimer's disease; VaD, vascular dementia. Based on Lobo et al. ${ }^{3}$

in women than in men and nearly doubled with every five year increase in age: $0.8 \%$ in the group age 65-69 years and $28.5 \%$ at age 90 years and older (fig 2). Of all dementia cases, $54 \%$ suffered AD. Prevalence of $\mathrm{AD}$ showed the steepest increase with age, from $0.6 \%$ in the group age $65-69$ years to $22.2 \%$ in the group aged 90 years and older. VaD accounted for $16 \%$ of cases, and prevalence increased with age from $0.3 \%$ (65-69 years) to $5.2 \%$ (90+ years). More recently, prevalence rates for dementia were compared among 12 population based European studies. Crude prevalence rates varied between $5.9 \%$ (Italy, the Counselice study) and $9.4 \%$ (the Netherlands, Rotterdam study). ${ }^{1}$ Again, an almost exponential increase with age and a female excess-mostly after age 75-was described.

\section{Dementia with young onset}

Most studies on prevalence of dementia focus on subjects aged over 65 years. Although age is well established as its most important risk factor, dementia may also affect people under the age of 65 . Few data exist on the prevalence of dementia in younger people. A recent study in the UK was designed to determine the prevalence of dementia in people under the age of 65 in a large catchment area (total population of 567500 people) and use these figures to estimate the number of younger people affected by dementia in the UK. ${ }^{4}$ The prevalence of dementia in those aged 30-64 was 54 per 100 000. For those aged 45-64, the prevalence was 98 per 100 000. Like the studies mentioned above describing prevalence over the age of 65, there was a strong age dependency: from the age of 35 years onwards, the prevalence of dementia approximately doubled with every

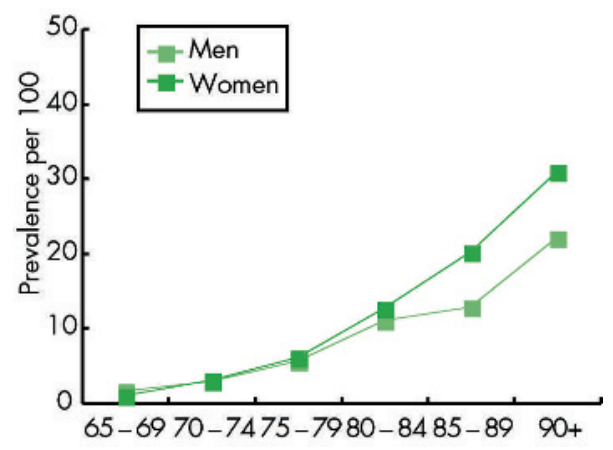

Figure 2 Pooled prevalence of dementia by sex. Based on Lobo et al. ${ }^{3}$ five year increase in age. In contrast with studies describing populations over 65 , males seem at a higher risk to become demented before they reach the age of 65 than females.

Extrapolating these figures nationally suggests that there are well over 18000 people with dementia under the age of 65 in the UK. At 34\%, AD was also the most prevalent cause of dementia among younger people, although with less prominence than at old age (fig 3 ). The relative prevalence of $\mathrm{VaD}(18 \%)$ roughly equals the prevalence at old age. Frontotemporal dementia (12\%) and alcohol related dementia $(10 \%)$ were relatively more prevalent among the younger population than among elderly populations. These figures underline the fact that, although relatively uncommon, dementia does develop in younger subjects, and it should always be part of the differential diagnosis in patients with cognitive complaints. Furthermore, these data also highlight the differences between dementia in younger people and dementia in older people, with frontotemporal dementia and alcohol related dementia being relatively common causes of dementia in the younger age group.

\section{"When I'm sixty four"}

Nearly 40 years ago, The Beatles launched a famous song which included the words "Will you still need me, will you still feed me when I'm 64". At that time Paul McCartney, looking at his 64 year old father, wondered how life would be at 64-considered "old" at that time apparently-probably also being afraid for age related diseases, such as dementia. In the coming decades, the financial and emotional burden placed by dementia on the working age population will rise notably. As the age distribution of the western population shifts, the rapid increase of the prevalence of dementia with increasing age means that both the number of affected individuals and the affected proportion of the total population are increasing. This will be especially prominent in Europe, where the median age of the population is higher than in all other parts of the world. Based on several metaanalyses of epidemiological studies and the population projections of the United Nations, the number of prevalent cases in Europe in the year 2000 was about seven million. ${ }^{5}$ Within the next 50 years, this number is estimated to more

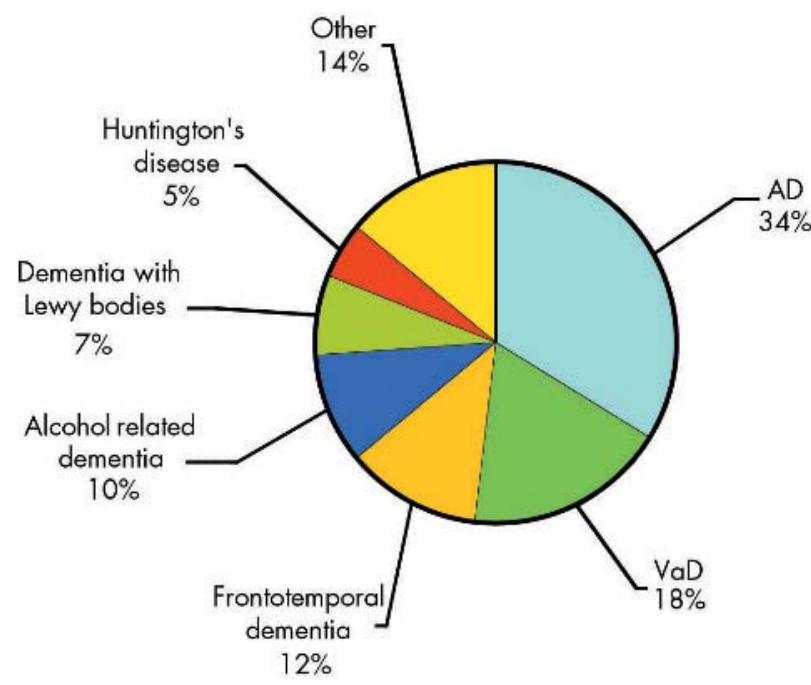

Figure 3 Causes of dementia with young onset ( $<65$ years). Based on Harvey et al. ${ }^{4}$ 


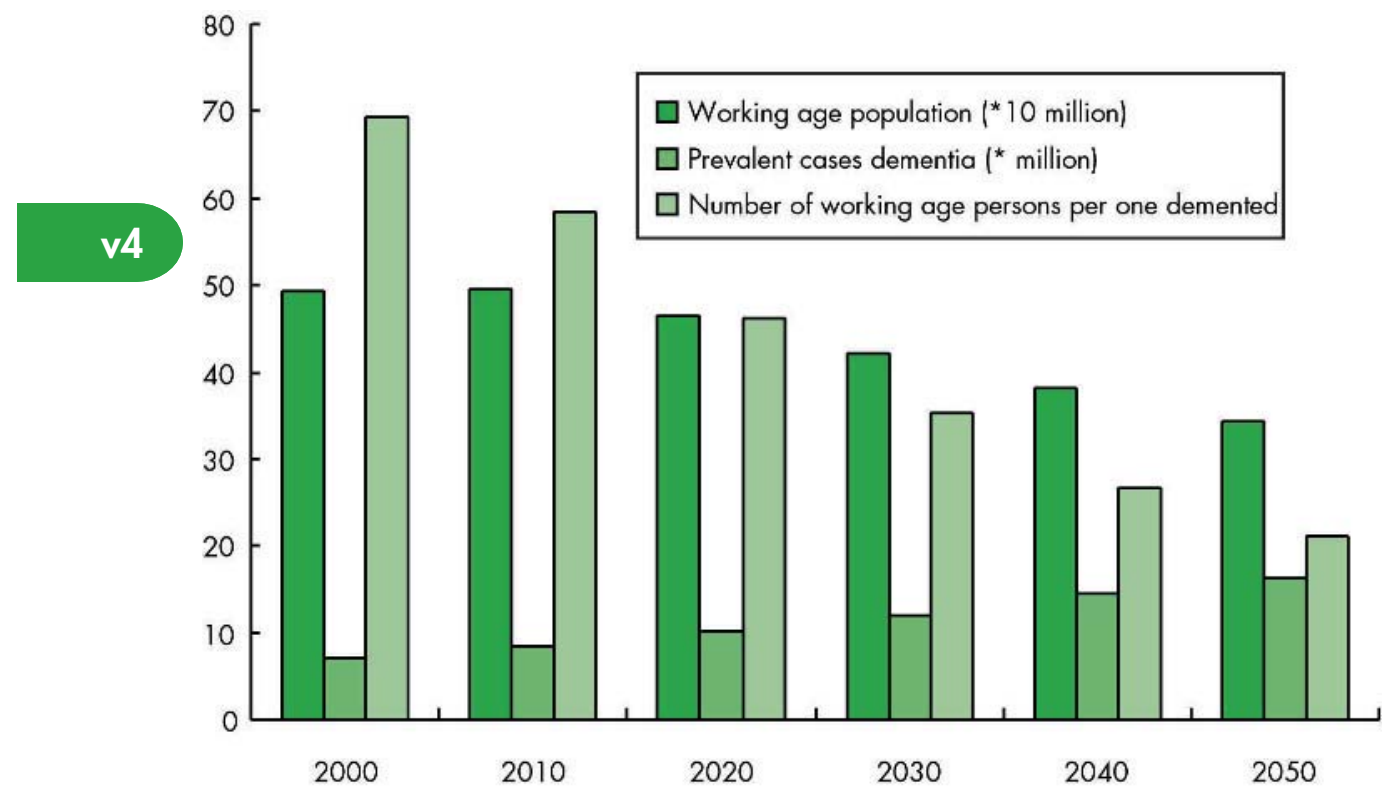

Figure 4 Working age population and number of working age persons per one demented person( $=$ ratio) in Europe using age and sex adjusted mean numbers of prevalent cases. Based on Wancata et al. ${ }^{5}$

than double to well over 16 million patients with dementia. Not only will the number of patients with dementia increase; in the same time span, the working age population will considerably decrease in number (fig 4). While in the year 2000, there was a ratio of 69 working age persons to one demented person, this ratio will decrease to $21: 1$ in 2050 .

In this paragraph, an overview of the prevalence of dementia has been given. Prevalence is determined by both the number of new cases over a given period of time, and by the duration of survival once patients have the disease. ${ }^{2}$ Death results in a decrease of prevalence; therefore, diseases that quickly lead to death may have low prevalence, even if they occur frequently, while diseases with long survival have higher prevalence, even if they occur with lower frequency. From the above it follows that studies based on prevalent cases yield associations that reflect the determinants of survival with disease just as much as the causes of disease. This can result in misleading situations-for example, if a new treatment would positively influence the course of dementia by lengthening survival (although not curing the disease), this would result in a higher prevalence. In such a situation, the paradoxical situation may occur that this medication would be positively associated with the prevalence of dementia, and so be misconstrued as a causative agent. For this reason incidence, rather than prevalence, is the desired measure of disease frequency.

\section{INCIDENCE}

Incidence refers to the number of new cases over a given period of time. The observed number of new cases depends on the evaluated duration of follow up. To be able to compare studies with varying duration of follow up, incidences per year are usually given. Furthermore, within a given study, length of follow up time may differ between subjects. To profit from all the available information, the length of time at risk is determined for every person. The total length of follow up time is obtained after summing all person-times, and represented as the number of person-years of follow up. Most studies on incidence report incidence rates that are calculated as the number of new cases divided by the person-years at risk. Incidence rates are usually represented as number of new cases per 1000 person-years.

\section{Pooled estimates of incidence}

In the same collaborative effort that pooled prevalence data of European studies, data on incidence of dementia of eight population based European studies were compared and pooled. ${ }^{6}$ In total, there were 42996 person-years of follow up with 835 new dementia cases. Of these, 60-70\% were diagnosed with $\mathrm{AD}$ and $15-20 \%$ with VaD. Incidence rates of dementia increased exponentially with age from 2.4 per 1000 person-years in the 65-69 age group, to 70.2 per 1000 personyears in the $90+$ age group. Rates among women were higher, especially above the age of 80 (fig 5 ). The rates continued to increase with age in women, whereas the increase reached a plateau in men at age 85 . For $\mathrm{AD}$, findings were comparable, with pooled incidence rates increasing from 1.2 per 1000 person-years among 65-69 year olds to 53.5 among subjects over 90 years old.

\section{Will we be all demented at the age of 140 ?}

The question of whether the incidence rates reach a plateau at a certain age is important, as an exponential increase in

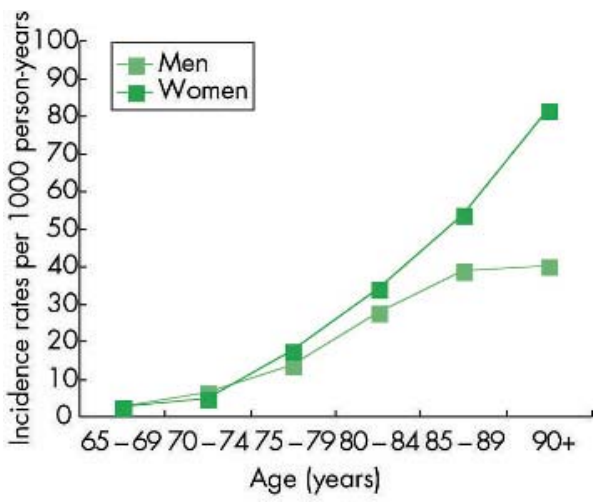

Figure 5 Pooled incidence rates of dementia by sex. Based on Fratiglioni et al. ${ }^{6}$ 
incident $\mathrm{AD}$ would suggest that the disease is an inevitable consequence of aging, whereas convergence to a fixed value or a decline could suggest that an element of the population has reduced vulnerability, owing perhaps to genetic or environmental factors. ${ }^{7}$ Results with respect to incidence increasing with age have been conflicting, with some studies suggesting an ongoing increase with advancing age, whereas other studies suggest that incidence rates reach a plateau after a certain age. This issue is difficult to resolve, however, as the oldest age groups are always underrepresented, resulting in less precise estimations. The Cache County in Utah, USA, is known for the longevity of its inhabitants. The relatively large proportion of extremely old individuals provides the opportunity to give reliable estimates of incident dementia among the oldest old. There were 185 new cases of dementia (123 AD) among 3308 participants who contributed 10541 person-years of follow up. ${ }^{7}$ The incidence of dementia increased with advancing age from 2 per 1000 person-years in the group aged $\leqslant 68$, to peak with 122 per 1000 person-years in the 90-92 age group, and decline in the $93+$ age group ( 110 per 1000 person-years). The incidence of dementia was higher in females over the age of 80 . If incidence rates would indeed plateau at a certain age, then the future public health burden of dementia and $\mathrm{AD}$, albeit still enormous, might be less than previously projected.

\section{Variation across regions?}

Incidence rates have been found to vary between studies. Methodological issues partly account for these differences, but it is also conceivable that the variable estimates reflect real geographical differences. There are substantial differences in possible risk factors for dementia between regions. Such chronic disease risk factor variation is thought to be responsible for the wide variation seen in other diseases of older age such as cancer and cardiovascular disease (for example, differences between North and South Europe). Given the available evidence for risk factors of dementia and the pronounced variation in vascular risk factors across regions, there could be parallel variation in the incidence of dementia. In fact, the pooled analysis of eight European studies mentioned above suggests a geographical dissociation, with higher incidence rates being found among the oldest old of northwestern countries than among southern countries. ${ }^{6}$ To assess variation in incidence within country, the Medical Research Council cognitive function and ageing study (MRC CFAS) compared incidence rates among five sites with different risk patterns and mortality rates. ${ }^{8}$ As reported before, incidence was observed to rise with age, particularly above the age of 75, and continued to increase for both males and females into the oldest age groups. However, there was no convincing evidence for variation across sites, and incidence rates did not reflect the variations in the prevalence of possible risk factors in these sites.

\section{RISK FACTORS}

The estimates of frequency of dementia are important by themselves, as they underline the extent of the health care problem as created by dementia. Although important for health care planners, the frequency of disease in itself is not the most important issue. Rather, we need to gain insight into the mechanisms that cause dementia, to be able to develop therapeutic agents that can slow down or even cure these diseases. Risk factors are studied to find out the basic mechanisms leading to dementia. By influencing these risk factors we hope to be able to modify the course of the disease.

Studies on risk factors for dementia have mainly focused on $\mathrm{AD}$, as it is the most frequent cause of dementia. Age is the most well known risk factor for dementia. Studies of prevalence and incidence of dementia and AD have consistently shown an almost exponential increase with advancing age, in that estimates of both prevalence and incidence double with every five year increase in age. In addition, female sex has repeatedly been shown to be associated with an increased risk of $\mathrm{AD}$, especially at old age. ${ }^{67}$ Other risk factors for $\mathrm{AD}$ include genetic and vascular factors.

\section{Genetic risk factors}

Only a small proportion of all individuals with dementia suffers from a familial form of dementia, caused by an autosomal dominant mutation. Mutations in several genes (including $\mathrm{A} \beta$ precursor protein, presenilin 1, and presenilin 2) have been shown to cause $A D$, but these genetic forms of $\mathrm{AD}$ account for less than $5 \%$ of all cases. The largest proportion of $\mathrm{AD}$ cases is therefore "sporadic". However, genetic factors also seem to influence non-familial cases of $\mathrm{AD}$. The "common disease/common variant" hypothesis postulates that common disorders, such as $\mathrm{AD}$, are also governed by common DNA variants. ${ }^{9}$ These variants significantly increase disease risk but are neither necessary nor sufficient to actually cause a specific disorder. Rather, these risk genes display intricate patterns of interaction with each other as well as with non-genetic variables, modifying the risk for a disease. To date, only one such factor has been identified in $\mathrm{AD}$. The apoliprotein $\mathrm{E}$ gene presents in three allelic forms $(\epsilon 2, \epsilon 3$, and $\epsilon 4)$, of which the $\epsilon 4$ allele is a risk factor for $\mathrm{AD} .{ }^{10} \mathrm{APOE} \in 4$ itself is neither necessary nor sufficient to cause $\mathrm{AD}$, but instead operates as a genetic risk modifier. The well known effect of age on $\mathrm{AD}$ is modified by APOE, as age of onset is lower in APOE $\epsilon 4$ positives. $^{7}$ In addition, it has been suggested that APOE interacts with vascular risk factors.

\section{Vascular risk factors}

There is abundant evidence that vascular factors play a role in AD. Vascular risk factors such as hypertension, diabetes mellitus, smoking, and heart disease all have been shown to be associated with AD. ${ }^{11}$ Explanations for these associations include: (1) the coincidence of common disorders in the elderly; (2) vascular and cerebrovascular disease precipitating $\mathrm{AD} ;(3)$ an additive or synergistic ( $\mathrm{AD}+$ vascular) pathogenesis of dementia; and (4) misclassification of vascular dementia as $\mathrm{AD} .^{11}{ }^{12}$ At this moment, the question about the primary and secondary pathology in $\mathrm{AD}$ is unlikely to be answered. The mechanisms linking vascular risk factors to $\mathrm{AD}$ remain unclear. Atherosclerosis has been postulated as one common mechanism mediating the association between $\mathrm{AD}$ and various vascular risk factors. However, statistical models have failed to demonstrate an important mediating role for atherosclerosis as one common factor. Either the measures of extracranial atherosclerosis are not suitable as proxies for intracranial atherosclerosis, or there are other mechanisms whereby cardiovascular risk factors are associated with $\mathrm{AD}$.

\section{METHODOLOGICAL ISSUES}

A brief overview has been given of the current knowledge of prevalence, incidence, and risk factors of dementia. Although 
progress in understanding dementia is being made, the basic mechanisms causing the majority of dementias are still not known, and satisfying therapeutic options are as yet not available. Studies of dementia are hampered by certain methodological issues inherent to the disorder. These methodological issues may influence the results of studies and be partly responsible for variability in results across studies. Without intending to give a complete overview of the methodological issues associated with the study of dementia, we would like to address briefly four important issues here.

\section{Diagnostic procedure}

The most important problem with respect to studying dementia and $\mathrm{AD}$ is defining the outcome. As yet, there is no single diagnostic test for $\mathrm{AD}$ or most of the other types of dementia. The diagnosis of $\mathrm{AD}$ is based on clinical criteria, and can be graded as possible, probable, or definite. Several sets of criteria are available, of which the criteria of the National Institute of Neurological and Communicative Disorders and Stroke (NINCDS) and the Alzheimer's Disease and Related Disorders Association (ADRDA), dating from 1984, are the most commonly used. ${ }^{13}$ The diagnostic work-up of dementia is time and cost intensive. In large population based studies, it is impossible to assess every subject with a complete diagnostic work-up. Using medical records to identify cases would lead to an underestimation of the number of individuals with dementia, as many of the cases of dementia are never diagnosed in a formal setting.

Therefore, large population based studies usually employ a stepwise approach to identify cases. Most studies use one of two possible stepwise approaches. (1) All subjects are assessed using a screening test. Only those performing below a certain cut off level receive an extensive assessment. A drawback of this approach is the low sensitivity of screening tests. Subjects who are demented but score above cut-off on the screening test are missed. These may include mild cases, and individuals with good cognitive reserve due to, for example, high educational level. (2) A subsample, stratified by certain characteristics such as age, sex, and performance on a screening test, receives an extensive diagnostic assessment. Results are extrapolated to the entire sample. Inherent to this approach is the fact that not all cases will receive an extensive assessment, which may result in lack of precision. The use of different criteria to diagnose dementia, and the variable approaches to operationalise these criteria in large samples, can result in highly varying estimates of frequency. The difficulty of diagnosing mild dementia can lead to an additional problem in incidence studies, as cases that are very mild and therefore not recognised at baseline may be wrongly counted as incident cases at follow up, resulting in biased estimates. $^{14}$

\section{Insidious onset}

A second-and related-methodological problem inherent to dementia is the insidious onset of the disorder. Neuropathological changes, eventually leading to the clinical syndrome of dementia, may start as early as decades before the disease becomes clinically overt. In analogy with the gradually accumulating neuropathology, the transition from healthy to demented is also gradual, rather than abrupt. The moment when dementia is diagnosed is in fact arbitrary. Moreover, the artificial dichotomisation between healthy and demented does not do justice to the continuum of cognitive (dys)function. The concept of mild cognitive impairment
(MCI) has been developed to account for the transitional phase between healthy and demented. ${ }^{15}$ However, introducing concepts like MCI only shifts the problem, as the borders between healthy and MCI and between MCI and demented remain just as arbitrary and unclear. A possible solution would be to discard the arbitrary distinction between normal and demented, and instead use a continuous outcome, such as a test of cognitive function. This would have several advantages. First, costs and time can be saved as the extensive diagnostic work-up is not necessary anymore. Second, by abolishing the artificial dichotomisation into normal and demented, the continuum of cognitive decline is done more justice. This approach also provides the opportunity to study progression of decline within demented individuals.

\section{Biomarkers}

A third issue reflects the complex relationship between the syndrome of dementia and the underlying diseases. When we talk of $\mathrm{AD}$, we refer to the syndrome that is characterised by progressive memory problems, which usually has an insidious onset, etc. However, at the moment the diagnosis of $\mathrm{AD}$ is made, we assume to know the underlying neuropathological substrate-that is, neuritic plaques and neurofibrillary tangles. We assume to know this, because during lifetime, it is impossible to directly measure neuropathology. In fact, postmortem studies have shown that this assumption in many cases is wrong. ${ }^{16}$ In a report of the MRC CFAS of the first 209 subjects (48\% demented) who came to necropsy, Alzheimertype pathology and vascular pathology were equally common, and both correlated with cognitive decline. Most subjects had mixed pathology. Approximately one third of clinically demented patients did not fulfil neuropathological criteria for definite $\mathrm{AD}$, whereas an equally large proportion of nondemented elderly subjects did fulfil these criteria. ${ }^{16}$ Neuropathologically, the distinction between different types of dementia, and even between demented and non-demented, seems to be very difficult. The question arises that if it is useful to make clinical distinctions between subtypes of dementia, neuropathology may not even exist. A step towards directly measuring disease, rather than clinical phenotype, would be to take biomarkers as outcome of studies. Both neuroimaging and cerebrospinal fluid can provide useful surrogate markers that give a more direct impression of the pathology. In this way, the possibility of different types of pathology coexisting within one subject is appreciated. For example, magnetic resonance imaging (MRI) measures suggestive of Alzheimer-type pathology and vascular pathology can be evaluated simultaneously.

\section{Cross sectional versus longitudinal studies}

Studies with a longitudinal design are preferred over studies with a cross sectional design for several reasons. It is conceivable that information about risk factors may be systematically different between patients and controls. Patient data must come from a proxy, who might recall the medical history differently than a proxy of a control or the control himself. In addition, prevalence is determined by both the number of new cases over a given period of time, and by the duration of survival once patients have the disease. In analogy, findings of cross sectional studies can reflect the contribution a risk factor makes to developing dementia as well as to surviving after the dementia starts. 
Another important issue in this respect is that risk factors may change over time. ${ }^{17}$ The impact of environmental factors, such as smoking, diet, physical activity, and vascular disease, may change over time both within an individual and across birth cohorts. Risk factors such as blood pressure change with ageing. Furthermore, the disease, once it has started, may in turn influence the risk factor. For example, the diet of a demented individual may change, when the person forgets to eat his or her meals on a regular basis. Therefore, the relationship between a risk factor and disease may differ depending on the age the risk factor is measured relative to the outcome.

The age related changes in risk factors make causal inferences as to the development of dementia difficult. Studies of blood pressure in relation to dementia form a good example of how the relationship between risk factors and dementia may be influenced by the moment when the risk factor is measured. ${ }^{12} 18$ There have been conflicting reports, with some studies suggesting that low blood pressure is associated with dementia, whereas others report the opposite, namely that high blood pressure is a risk factor for dementia. Important in this respect is that blood pressure has been shown to decrease as a consequence of dementia. It is therefore important that this risk factor (blood pressure) is measured before the disease process starts. However, by the age most ageing studies begin-that is, 65 years oldindividuals have already experienced the initial neuropathologic changes that eventually lead to dementia. As soon as the disease process has started (this may be years, possibly decades, before the dementia becomes overt), it is too late to measure risk factors, as the disease may have started to influence the risk factor itself.

Therefore, it seems as though risk factors should be measured as early as possible. By now, there are several studies with more than 20 years of follow up. ${ }^{19-21}$ These studies, measuring midlife risk factors to predict late life dementia, have shed some light on the perceived incongruence in earlier studies. In fact, the conflicting reports with respect to the effect of blood pressure on the development of dementia may be entirely explained by the moment of measuring the risk factor. Cross sectional studies suggest that low blood pressure is associated with dementia. Studies measuring blood pressure during midlife have consistently shown that midlife hypertension is associated with late life dementia.

\section{CONCLUSIONS}

- A review of the epidemiology of dementia indeed reveals that the public health problem of dementia has reached epidemic proportions. It affects about $6 \%$ of individuals over 65 years of age and has a strong age dependent prevalence. Although dementia is quite rare before 65, it certainly does occur, and dementia should always be in the differential diagnosis when evaluating patients with cognitive complaints, irrespective of age. $\mathrm{AD}$ is the most prevalent form of dementia, responsible for about $60-70 \%$ of cases. VaD is the second most important cause, accounting for $15-20 \%$.

- In the age group 65-69 years, there are more than two new cases per 1000 persons, every year. This number increases almost exponentially with increasing age, until over the age of 90 years, out of 1000 persons, 70 new cases of dementia can be expected every year.

- Risk factors for dementia include age and the female sex, especially at high age. Moreover, genetic factors (APOE $€ 4)$ and vascular risk factors play an important role.

- Methodological problems associated with studies of dementia include (1) complex diagnostic procedure, (2) insidious onset, and (3) the relationship between the clinical syndrome and the disease as defined by the underlying neuropathology. Finally, it is argued that longitudinal studies are to be preferred over cross sectional studies.

\section{Authors' affiliations}

W M van der Flier, P Scheltens, Department of Neurology and Alzheimer Center, Vrije Universiteit Medical Center, Amsterdam, the Netherlands

\section{REFERENCES}

1 Berr C, Wancata J, Ritchie K. Prevalence of dementia in the elderly in Europe. Eur Neuropsychopharmacol 2005; 15:463-71.

2 Rothman K, Greenland S. Modern epidemiology, 2nd ed. Philadelphia: Lippincott-Raven, 1998.

3 Lobo A, Launer $U$, Fratiglioni L, et al. Prevalence of dementia and major subtypes in Europe: a collaborative study of population-based cohorts. Neurologic diseases in the elderly research group. Neurology 2000;54(1) suppl 5):S4-9.

4 Harvey RJ, Skelton-Robinson M, Rossor MN. The prevalence and causes of dementia in people under the age of 65 years. J Neurol Neurosurg Psychiatry 2003;74:1206-9.

5 Wancata J, Musalek M, Alexandrowicz R, et al. Number of dementia sufferers in Europe between the years 2000 and 2050. Eur Psychiatry 2003; 18:306-13.

6 Fratiglioni L, Launer $\sqcup$, Andersen K, et al. Incidence of dementia and major subtypes in Europe: a collaborative study of population-based cohorts. Neurologic diseases in the elderly research group. Neurology 2000;54(11 suppl 5):S10-15.

7 Miech RA, Breitner JC, Zandi PP, et al. Incidence of AD may decline in the early 90 s for men, later for women: the Cache County study. Neurology 2002;58:209-18.

8 Matthews F, Brayne C, Investigators MR. The incidence of dementia in England and Wales: findings from the five identical sites of the MRC CFA Study. PLoS Med 2005;2:e193.

9 Lander ES. The new genomics: global views of biology. Science 1996;274:536-9

10 Strittmatter WJ, Roses AD. Apolipoprotein E and Alzheimer disease. Proc Natl Acad Sci U S A 1995;92:4725-7.

11 Breteler MM. Vascular risk factors for Alzheimer's disease: an epidemiologic perspective. Neurobiol Aging 2000;21:153-60.

12 Launer LJ. Demonstrating the case that $A D$ is a vascular disease: epidemiologic evidence. Ageing Res Rev 2002;1:61-77.

13 McKhann G, Drachman D, Folstein M, et al. Clinical diagnosis of Alzheimer's disease: report of the NINCDS-ADRDA work group under the auspices of Department of Health and Human Services task force on Alzheimer's disease. Neurology 1984;34:939-44.

14 Launer LJ, Brock DB. Population-based studies of AD: message and methods: an epidemiologic view. Stat Med 2004;23:191-7.

15 Petersen RC, Smith GE, Waring SC, et al. Mild cognitive impairment: clinical characterization and outcome. Arch Neurol 1999;56:303-8.

16 MRC CFAS Investigators. Pathological correlates of late-onset dementia in a multicentre, community-based population in England and Wales. Neuropathology group of the Medical Research Council cognitive function and ageing study (MRC CFAS). Lancet 2001;357:169-75.

17 Launer LJ. The epidemiologic study of dementia: a life-long quest? Neurobiol Aging 2005;26:335-40.

18 Qiu C, Winblad B, Fratiglioni L. The age-dependent relation of blood pressure to cognitive function and dementia. Lancet Neurol 2005;4:487-99.

19 Kivipelto M, Helkala EL, Laakso MP, et al. Apolipoprotein E epsilon4 allele, elevated midlife total cholesterol level, and high midlife systolic blood pressure are independent risk factors for late-life Alzheimer disease. Ann Intern Med 2002; 137:149-55.

20 Launer $\mathrm{L}$, Ross GW, Petrovitch $\mathrm{H}$, et al. Midlife blood pressure and dementia the Honolulu-Asia aging study. Neurobiol Aging 2000;21:49-55.

21 Korf ES, White LR, Scheltens P, et al. Midlife blood pressure and the risk of hippocampal atrophy: the Honolulu Asia aging study. Hypertension 2004;44:29-34. 